\title{
A solid-state NMR perspective of the hydrogen atom location and energetics in low-barrier hydrogen bonds
}

\author{
Gang Wu \\ Department of Chemistry, Queen's University, Kingston, Ontario, Canada K7L \\ 3N6,wugang@queensu.ca
}

A low-barrier hydrogen bond ( $\mathrm{LBHB}$ ) is commonly defined as a case where the energy barrier for the hydrogen atom to shuttle between the two heavy atoms involved in the HB becomes comparable to the zero-point energy [1]. Conceptually, a LBHB seems to be just an ordinary intermediate case between a very strong $\mathrm{HB}$, where the potential is a single-well, and a weak HB, where the potential corresponds to a highly asymmetrical double-well. However, the designation of a new name (i.e., LBHB) is too often taken automatically by most people as a hint that this class of HBs are special. In the past three decades, an intense debate has centered on the role of LBHBs in enzymatic reactions [2-10]. While structural and spectroscopic characteristics of LBHBs are well documented in the literature, several aspects of LBHBs are yet to be completely understood. For example, how important is it to consider the delocalization of the $\mathrm{H}$ atom (or more precisely the nuclear wavefunction) in LBHBs? What is the energetics of a LBHB? What can one learn about LBHBs from solid-state NMR studies? In this talk, I will present new solid-state NMR results to shed light on some fundamental aspects of LBHBs.

\section{References}

[1] Hibbert, F. \& Emsley, J. (1990). Adv. Phys. Org. Chem., 26, 255-379.

[2] Cleland, W.W. (1992). Biochemistry, 31, 317-319.

[3] Gerlt, J.A. \& Gassman, P.J. (1993). J. Am. Chem. Soc., 115, 11552-11568.

[4] Perrin, C.L. \& Nielson, J.B. (1997). Annu. Rev. Phys. Chem., 48, 511-544.

[5] Cleland, W.W. \& Kreevoy, M.M. (1994). Science, 264, 1887-1890.

[6] Warshel, A. et al. (1995). Science, 269, 102-104.

[7] Scheiner, S. \& Kar, T. (1995). J. Am. Chem. Soc., 117, 6970-6975.

[8] Guthrie, J.P. (1996). Chem. Biol., 3, 163-170.

[9] Warshel, A. \& Papazyan, A. (1996). Proc. Natl. Acad. Sci. USA, 93, 13665-13670.

[10] Perrin, C.L. (2010). Acc. Chem. Res., 43, 1550-1557. 